\title{
Challenge in challenging times: a case report of ruptured isthmic ectopic pregnancy in an asymptomatic COVID-19 positive patient
}

\author{
Ankita Gahlot, Atri Raval*, Bindoo Yadav, Swarnima Saxena
}

Department of Obstetrics and Gynaecology, SGT Medical College Hospital and Research Institute, Gurgaon, Haryana, India

Received: 26 July 2021

Accepted: 04 September 2021

\section{*Correspondence:}

Dr. Atri Raval,

E-mail: anujraval26@gmail.com

Copyright: () the author(s), publisher and licensee Medip Academy. This is an open-access article distributed under the terms of the Creative Commons Attribution Non-Commercial License, which permits unrestricted non-commercial use, distribution, and reproduction in any medium, provided the original work is properly cited.

\begin{abstract}
Emergency surgical management of patients who are COVID-19 positive is extremely challenging for the treating doctors as there is a need to keep the balance between delivering optimal medical care to the patient, preventing spread to others and protecting ourselves. COVID-19 manifestations in pregnant women are similar to non-pregnant patients. The patient may be asymptomatic or may present with symptoms like cough, cold, fever and shortness of breath. In obstetric emergencies like ruptured ectopic pregnancy, when a patient presents in casualty with history of amenorrhea, pain abdomen and features of shock, main priority for the clinician is to stabilize them and treat the underlying cause so as to save their life. We report the emergency management of a hemodynamically unstable ruptured ectopic pregnancy at 9 weeks of gestation in a COVID-19 positive patient. It was stressful for the treating doctors because of high infectivity of the disease, scarcity of resources during the pandemic and precarious condition of the patient.
\end{abstract}

Keywords: Ruptured ectopic, COVID-19, Obstetrics emergency

\section{INTRODUCTION}

Incidence of ectopic pregnancy in Haryana is $1.48 \%$ of all pregnancies. ${ }^{1}$ Ampullary ectopic is the most common accounting to $80-90 \%$ of all ectopic pregnancies while Isthmic accounts for $15.3 \% .^{2}$ Tubal ectopic pregnancy has high chances of rupture in early pregnancy because the rapidly proliferating trophoblasts erode the muscular layer of fallopian tube causing haemorrhage. If not detected timely this can have grave consequences with a significant high morbidity and mortality. Incidence of maternal deaths due to ectopic pregnancy has been reported to be 3.5$7.1 \% .^{3}$ Technological advancement has helped in early diagnosis of ectopic pregnancy before rupture thereby reducing the mortality due to the same. ${ }^{4}$ These patients can be offered medical treatment or at times expectant management if detected before rupture.

In order to prevent the spread of COVID-19 disease restrictive measures like nationwide lockdown have been taken. This trepidation about getting COVID-19 infection has led to deleterious effects on public health, possibly due to reluctance in seeking medical advice. There has been a decline in number of visits to the hospital for routine check-up. Dvash et al reported increased incidence of ruptured ectopic pregnancy at their centre in this pandemic. 5

\section{CASE REPORT}

A 34-year-old primigravida presented in obstetrics casualty with acute onset pain abdomen and shortness of breath. Patient gave history of amenorrhoea for 2 months. There was no history of fever, cough or bleeding per vaginum. Patient had no investigations like pregnancy test or ultrasonography scan. On examination radial pulse and blood pressure were not recordable, $\mathrm{SpO}_{2}$ was $98 \%$ at room air. She was very pale and was stabilized by transfusing IV fluids and colloidal agents. Patient was catheterized and urine for pregnancy test was done. The 
positive report raised high suspicion of ruptured ectopic pregnancy.

On abdominal examination tenderness was present in right iliac fossa. Per-vaginal examination cervical motion tenderness present, fullness felt in right fornix. On the basis of history and clinical examination provisional diagnosis of ruptured ectopic pregnancy in hypovolaemic shock was made. The need for blood transfusion and testing for COVID-19 was explained to them. In view of the poor general condition of the patient, decision for urgent exploratory laparotomy was taken without waiting for report of COVID-19 status.

In the operation theatre, under standard monitoring and use of full personal protection equipment by the health care workers involved, emergency exploratory laparotomy was performed under general anaesthesia. The intubation was done by a trained anaesthetist. Per operatively the ruptured gestation-sac was identified in right isthmic part of tube. There was significant hemoperitoneum and conclusive diagnosis of ruptured ectopic pregnancy was made. Use of electro cautery was avoided at all times. ${ }^{6}$ Right salpingectomy was performed and haemostasis was secured after separating the gestation sac from the isthmic portion of tube. Intra operative blood transfusion was started. Estimated blood loss during surgery was around $1500 \mathrm{ml}$. Standard waste disposal protocols were followed post-surgery.

Post operatively patient was shifted to COVID-19 ward as she was found to be RAT positive and subsequently RTPCR positive next day. She was managed post operatively according to standard COVID-19 guidelines. Post-operative period was uneventful and patient was discharged early. ${ }^{7}$

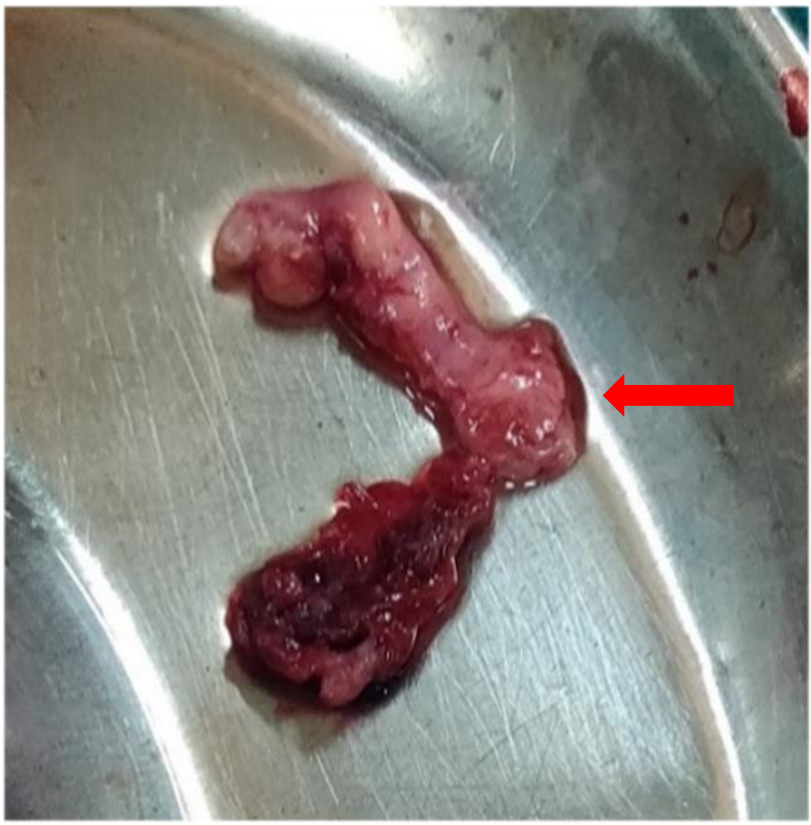

Figure 1: Right side fallopian tube.

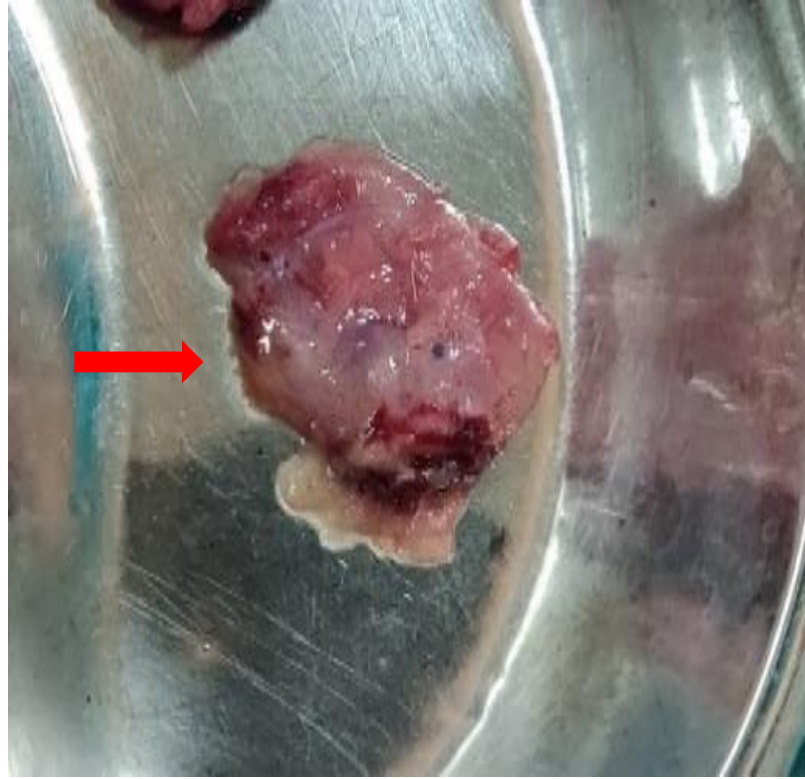

Figure 2: Gestational sac (complete).

\section{DISCUSSION}

With rise in COVID-19 cases and country wide lockdown, there is an innate fear and reluctance in people to go out and avail medical facilities. Goyal et al reported that delay in seeking health care advice due to lockdown and fear of contracting infection resulted in $44.7 \%$ of pregnancies with complications. ${ }^{8}$ This has taken a toll on emergent nonCOVID-19 related visits to the emergency department and, as such, on non-COVID-19-related deaths. ${ }^{9}$ A similar case as above could have been detected at an early gestation in non- COVID era by routine ultrasonographic scan and managed timely medically without any emergency surgical intervention. For patients with COVID-19 and ectopic pregnancy in vitally stable conditions (i.e., no rupture), medical therapy with methotrexate is preferable.

Significance of knowing the exact location of the ectopic, especially in these COVID times, was highlighted by the latest international society of ultrasound in obstetrics and gynaecology consensus statement. ${ }^{10}$ Barg et al have reported that in this pandemic of COVID-19 more women have reported after tubal rupture than before rupture thereby increasing need for emergency surgical management and blood transfusion. ${ }^{11}$

In our hospital a policy was made to all elective deferrable surgeries were cancelled, while acute emergencies were managed. The existing health care infrastructure, which was already burdened in non-COVID period was further divided between COVID and non-COVID patients. There was limited supply of equipment and consumables like oxygen for non-COVID patients.

In emergency conditions time becomes the essence of great importance. Our hospital has in-house SARS-CoV-2 reverse transcription polymerase chain reaction testing but 
confirmatory results could take a few hours. Waiting for COVID-19 test result could have led to delay in undertaking the procedure. On the other hand, immediate surgery could put staff and other patients at risk if patient tested positive for infection. Hence patient was presumed positive and laparotomy was performed keeping in view the COVID-19 guidelines so as to minimize risk of spread to staff and other patients.

\section{CONCLUSION}

In last one and half years since the first case was reported at Wuhan there has been a steady increase in our knowledge of COVID-19 infection and the disease. However, the restrictions imposed have resulted in women coming reluctantly for check-up. Health care providers will need to be proactive in dealing with avoidable lifethreatening complications like ruptured ectopic pregnancy. Common surgical procedures may have to be modified in order to contain the infection like limited use of electrocautery, all health care workers should be trained in use of personal protective equipment (PPE) kits and aware of guidelines. In this case clinical acumen and preparedness helped in managing the ruptured ectopic pregnancy in shock.

\section{Funding: No funding sources}

Conflict of interest: None declared

Ethical approval: Not required

\section{REFERENCES}

1. Chaudhary S, Chauhan MB, Gupta A, Dalal M. Clinical study of ectopic pregnancy at tertiary care center in Haryana, India. Int J Reprod Contracept Obstet Gynecol. 2019;8(7):2816.

2. Homm RJ, Holtz G, Garvin A. Isthmic ectopic pregnancy and salpingitis isthmic nodosa. Fertility Sterility. 1987;48(5):756-60.

3. Yadav K, Namdeo A, Bhargava M. A Retrospective and prospective study of maternal mortality in a rural tertiary care hospital of central India. Indian $\mathrm{J}$ Community Health. 2013;25(1):16-21.
4. Lipscomb GH. Ectopic Pregnancy. Obstetri Gynecol. 2010;115(3):487-8.

5. Dvash S, Cuckle H, Smorgick N, Vaknin Z, Padoa A, Maymon R. Increase rate of ruptured tubal ectopic pregnancy during the COVID-19 pandemic. Eur J Obstet Gynecol Reprod Biol. 2021;259:95-9.

6. Karuppal R, Surendran S, Patinharayil G, Muhammed Fazil VV, Marthya A. It is time for a more cautious approach to surgical diathermy, especially in COVID19 outbreak: A schematic review. J Orthop. 2020;20:297-300.

7. De Simone B, Chouillard E, Sartelli M, Biffl WL, Di Saverio S, Moore EE et al. The management of surgical patients in the emergency setting during COVID-19 pandemic: the WSES position paper. World J Emerg Surg. 2021;16:1.

8. Goyal M, Singh P, Singh K, Shekhar S, Agrawal N, Misra S. The effect of the COVID-19 pandemic on maternal health due to delay in seeking health care: Experience from a tertiary center. Int $\mathbf{J}$ Gynecol Obstet. 2021;152(2):231-5.

9. Anteby M, Van Mil L, Michaan N, Laskov I, Grisaru D. Effects of the COVID-19 pandemic on timely care for extrauterine pregnancies: A retrospective analysis. Lancet Regional Health-Eur. 2021;2:100026.

10. Royal College of Obstetricians and Gynaecologists. Guidance for antenatal screening and ultrasound in pregnancy in the evolving coronavirus (COVID-19) pandemic. Available at: https://www.rcog.org.uk/globalassets/documents/gui delines/2020-03-25-covid19-ntenatalscreening.pdf(2020). Accessed on 10 Feb 2021.

11. Barg M, Rotem R, Mor P, Rottenstreich M, Khatib F, Grisaru-Granovsky $\mathrm{S}$ et al. Delayed presentation of ectopic pregnancy during the COVID-19 pandemic: A retrospective study of a collateral effect. Int $\mathbf{J}$ Gynaecol Obstet. 2021;153(3):457-61.

Cite this article as: Gahlot A, Raval A, Yadav B, Saxena S. Challenge in challenging times: a case report of ruptured isthmic ectopic pregnancy in an asymptomatic COVID-19 positive patient. Int J Reprod Contracept Obstet Gynecol 2021;10:3976-8. 